\title{
On-line Identification of Vehicle Fuel Consumption for Energy and Emission Management: An LTP System Analysis
}

\author{
J.T.B.A. Kessels, J. Sijs, R.M. Hermans, A.A.H. Damen, P.P.J. van den Bosch \\ Technische Universiteit Eindhoven \\ Department of Electrical Engineering \\ P.O. Box 513, 5600 MB Eindhoven, The Netherlands \\ E-mail: J.T.B.A.Kessels@tue.nl; P.P.J.v.d.Bosch@tue.nl
}

\begin{abstract}
An Energy Management (EM) system traditionally relies on (quasi) static maps offering efficiency parameters of the vehicle powertrain. During a vehicle's life span, these maps lose validity, so optimal performance for EM is not assured. This paper presents a proof-of-concept for a novel measurement system, estimating important engine and generator characteristics on-line during driving.

The generator applies a small excitation signal to the combustion engine and by means of correlation techniques and feedback control, the incremental fuel cost for generating electric power is estimated. This information is very relevant for EM in Hybrid Electric Vehicles. No additional sensors (e.g. torque estimators) are needed.

Under mild assumptions it is shown that the measurement system satisfies a Linear Time Periodic (LTP) System. Harmonic analysis as well as Floquet Theory are used to analyze performance and stability criteria. Simulation results support this analysis and demonstrate good noise rejection of the system.
\end{abstract}

\section{INTRODUCTION}

Owing to the high standards on safety and comfort, the electric power demand of today's vehicles increases rapidly. Simultaneously, the automobile industry is forced to reduce their fleet emissions due to environmental regulations. As a consequence, innovative research appears in the field of advanced power supply systems [12] and emission aftertreatment [1], as well as alternative vehicle concepts, e.g., Hybrid Electric Vehicles (HEVs) or Hydrogen Vehicles. Energy \& Emission Management (EM) is needed to supervise the vehicle power flow and satisfy its power demand in a fuel efficient and environmental friendly way [2].

An EM strategy requires knowledge about the vehicle status and its surrounding to decide upon production, storage and consumption of energy. Existing strategies rely on vehicle models, with parameters derived from static maps of components [9]. In general, these static maps originate from experiments within a conditioned test environment. However, road vehicles are subject to many operational conditions and transients appear during acceleration and deceleration phases. Moreover, vehicles suffer from wear and periodic service is needed to guarantee reliable operation. It is clear that regular updates of model parameters are necessary to guarantee robust performance for EM. Ultimately, on-line measurement and adaptation of these parameters is foreseen.

To maximize the performance of existing EM strategies, this paper presents a novel measurement system for on- line identification of powertrain's specific fuel consumption. Regarding the identification of Internal Combustion Engines (ICE), most work concentrates on estimating engine torque [8], [10] and almost no attention is paid to estimating the ICE fuel consumption, rather than using expensive mass flow sensors for on-line measurement. The electric generator is separately identified on a test-bench. In this paper, identification of both the ICE and the generator is done simultaneously through a combination of signal correlation and control. The system utilizes the generator of the vehicle to apply a (small) periodic disturbance on the output power of the primary power source, the ICE. Initially, the ICE exhibits a small perturbation on its nominal speed, but by means of correlation and non-linear feedback, the amplitude of the perturbation is estimated and compensated via the fuelinjection signal. This provides enough information to derive the incremental fuel costs for generating electric power.

Besides benefits for EM, the proposed system offers many extra advantages. It can be used to detect when a vehicle needs to be serviced, so maintenance costs are minimized. Also variations in fuel quality are recognized and can be taken into account by the engine control unit.

Although the measurement system is still in development, simulation results demonstrate that the measurement concept is valid. In this paper, emphasis is put on system analysis. It is shown how the non-linearities, arising from two multiplications in the correlation method, can be represented as a Linear Time Periodic (LTP) system. Various performance and stability aspects are elaborated using Harmonic analysis and Floquet's theory, including a proof of stability.

The structure of this paper is as follows. An overview of the measurement system is given in Section II. A system analysis is done in Section III and IV, using Harmonic Analysis. Numerical stability is addressed in Section V by means of Floquet's theory. Section VI shows preliminary results from simulations and the conclusions are drawn in Section VIII.

\section{SYSTEM DESCRIPTION}

The measurement system identifies the additional fuel use $\Delta f[\mathrm{~g} / \mathrm{s}]$ of the Internal Combustion engine (ICE) for producing additional electric power $\Delta P$ [W] with the generator. A small disturbance $\Delta P$ is added to the generator 


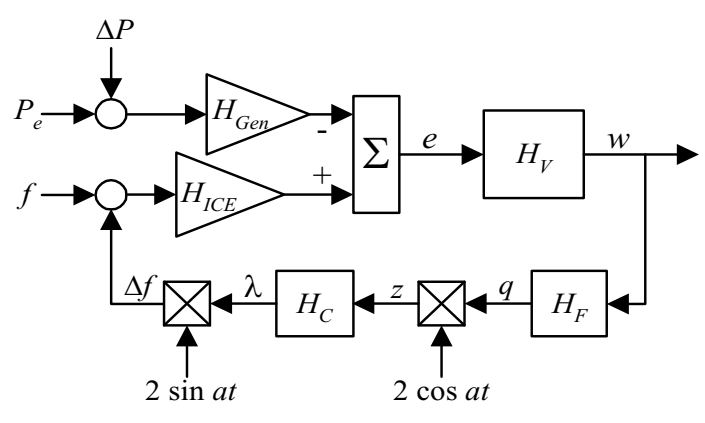

Fig. 1. Block diagram for system identification

power and the fuelling system is used to compensate for this disturbance by means of fuel injection $\Delta f$. In case the identification is done with an infinite small disturbance, the incremental fuel cost $\lambda$ can be deduced for a given operating point $\theta$ :

$$
\lambda(\theta)=\left.\left.\frac{\partial f}{\partial P}\right|_{\theta} \approx \frac{\Delta f}{\Delta P}\right|_{\theta} \quad[\mathrm{g} / \mathrm{J}]
$$

Hence, $\lambda(\theta)$ expresses the amount of additional fuel $\Delta f$ to produce $\Delta P$ additional electric power. To apply EM in HEVs, this information is crucial since it directly tells when to produce electric power (i.e. where $\lambda(\theta)$ reaches its minimum), see [5], [6]. Although $\lambda(\theta)$ originates from Lagrange multipliers, other related work denotes this quantity as Fuel Specific Equivalent, as it describes the equivalent fuel mass flow for a specific power increase, see e.g., [4], [11]. It is foreseen that $\theta$ covers many variables, amongst others, the engine speed, engine torque, temperature, generator power. In this work, however, a proof-of-concept will be given for one fixed operating point $\theta$.

\section{A. Correlation method}

A block diagram of the measurement system is shown in Fig. 1. The inputs $f[\mathrm{~g} / \mathrm{s}]$ and $P_{e}[\mathrm{~W}]$ represent the driver's request for engine power and alternator power when driving a certain speed. A sinusoidal signal with frequency $a[\mathrm{rad} / \mathrm{s}]$ is injected for system identification, so $\Delta P=2 \sin (a t)$. The ICE and the generator can be seen as a gain, which needs to be identified. The generator is mechanically connected to the engine's crankshaft, and the $\Sigma$-block demonstrates how its torque request is subtracted for the ICE torque. The remaining torque, expressed by $e[\mathrm{Nm}]$ in Fig. 1, becomes available for vehicle propulsion. The dynamic behavior of the vehicle is covered by the transfer function $H_{V}(s)$. Only the engine speed $w$ [rad/s] is used by the measurement system.

Without feedback, two frequency components are recognized in the engine speed: $w=w_{V}+\Delta w$. The first constant term $w_{V}$ emerges from the constant vehicle speed whereas the second frequency is the known disturbance with frequency $a$. For compensating the disturbance $\Delta w$, the measurement system entails a feedback path, applying the following correlating actions:

1) A bandpass filter $H_{F}$ selects the signal $\Delta w$ from $w=$ $w_{V}+\Delta w$.
2) Demodulation of $\Delta w$ through multiplication with $u_{D}(t)=2 \cos (a t)$. The signal $\Delta w$ maps on two sidebands with the frequencies $\omega_{1}=0$ and $\omega_{2}=2 a$.

3) A controller $H_{C}$ reduces $\Delta w$ by means of an integrator. This yields $\lambda$.

4) Modulation of $\lambda$ with $u_{M}(t)=2 \sin (a t)$ leads to the desired compensation $\Delta f$.

Due to multiplication with sinusoidal signals, a non-linear feed-back path emerges. This looks similar to the feedback scheme from Extremum Seeking (see e.g., [13] and the references therein), but is different since two multiplications are now used.

In steady state, there holds $z=0$ so $\Delta w=0$. For this operating point, $\lambda$ equals the quotient of the generator gain $H_{G e n}(s)=K_{G e n}$ and the ICE gain $H_{I C E}(s)=K_{I C E}$ :

$$
K_{G e n} \Delta P=K_{I C E} \Delta f \Rightarrow \lambda=\frac{\Delta f}{\Delta P}=\frac{K_{G e n}}{K_{I C E}} .
$$

This quotient is a good measure of the conversion from (additional) fuel into (additional) electric power from (1).

\section{B. Linear periodic model}

To evaluate stability and performance characteristics of the measurement system, the vehicle model $H_{V}$ is reduced and linearized around a fixed engine speed $w_{V}$. This yields a first order model between the input torque $E(s)$ and the engine speed $W(s)$ :

$$
H_{V}(s)=\frac{W(s)}{E(s)}=\frac{1}{J s+d},
$$

with $J\left[\mathrm{kgm}^{2}\right]$ the equivalent inertia and $d[\mathrm{Nms} / \mathrm{rad}]$ the damping. $H_{F}(s)$ is a second order bandpass filter (critically damped at frequency $a$ ):

$$
H_{F}(s)=\frac{Q(s)}{W(s)}=\frac{a^{2} s}{(s+a)^{2}} .
$$

For the controller $H_{C}(s)$ an integrator has to be used:

$$
H_{C}(s)=\frac{\Lambda(s)}{Z(s)}=\frac{K}{s},
$$

with $K$ the gain of the controller. It will be shown that this gain is important for stability and performance of the system. For simplicity, the ICE gain and the generator gain are selected fixed $\left(K_{I C E}=K_{G e n}=1\right)$, although they depend on $\theta$ in reality. The reduced system is redrawn in Fig. 2, using positive feedback:

$$
E(s)=-R(s)+\Delta F(s) .
$$

The system shown in Fig. 2 satisfies a Linear Time Varying (LTV) system with the state-space description:

$$
\begin{aligned}
& \dot{x}(t)=A(t) x(t)+B(t) u(t) \\
& y(t)=C(t) x(t)+D(t) u(t)
\end{aligned}
$$

with state vector $x=\left[\begin{array}{llll}w & q_{1} & q_{2} & \lambda\end{array}\right]^{\top}$ and

$$
A(t)=\left(\begin{array}{cccc}
-\frac{d}{J} & 0 & 0 & \frac{2}{J} \sin (a t) \\
a^{2} & -2 a & -a^{2} & 0 \\
0 & 1 & 0 & 0 \\
0 & 2 K \cos (a t) & 0 & 0
\end{array}\right)
$$




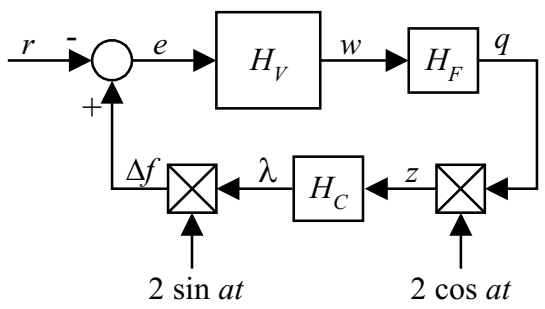

Fig. 2. Simplified measurement system

Selecting input $u=r$ and output $y=\lambda$ yields $B=$ $\left[\begin{array}{llll}-\frac{1}{J} & 0 & 0 & 0\end{array}\right]^{\top}, C=\left[\begin{array}{llll}0 & 0 & 0 & 1\end{array}\right]$ and $D=0$. Note that the system (7) is $T$-periodic, because there holds:

$$
A(t+T)=A(t)
$$

for all $t$. Therefore, the system is also addressed as a Linear Time Periodic (LTP) system.

\section{HARMONIC ANALYSIS}

Although the used correlation method with sinusoidal multiplication is indeed very selective, it does not only compensate for $\Delta P$, but it also injects new signals with frequency $n a, n \in \mathbb{Z}$ into the system. To explain the relationship between all individual frequency components, this section considers the Harmonic Analysis as studied by Wereley and Hall [15]. This method will also be used to analyse performance characteristics of the system in Section VI.

Definition 1 (Exponentially Modulated Periodic signal [15]): An Exponentially Modulated Periodic (EMP) signal can be expressed as the complex Fourier series of a periodic signal of frequency $\omega_{p}$, modulated by a complex exponential signal,

$$
u(t)=\sum_{n \in Z} u_{n} e^{s_{n} t}
$$

where $t \geq 0, s_{n}=s+j n \omega_{p}$, and $s \in \mathbb{C}$.

Assigning an EMP signal $u(t)$ to the LTP system (7) implies that the steady state response is also EMP,

$$
\begin{aligned}
& x(t)=\sum_{n \in Z} x_{n} e^{s_{n} t}, \\
& \dot{x}(t)=\sum_{n \in Z} s_{n} x_{n} e^{s_{n} t},
\end{aligned}
$$

and also the output $y(t)$ is an EMP signal

$$
y(t)=\sum_{n \in Z} y_{n} e^{s_{n} t} .
$$

The dynamics matrix (8) has fundamental frequency $\omega_{p}=$ $a$ and using Euler's formula, it can be expanded in a complex Fourier series

$$
\begin{aligned}
A(t) & =\sum_{m \in \mathbb{Z}} A_{m} e^{j m \omega_{p} t} \\
& =A_{-1} e^{-j \omega_{p} t}+A_{0}+A_{1} e^{j \omega_{p} t}
\end{aligned}
$$

with Fourier coefficients $A_{m}$ :

$$
\begin{gathered}
A_{0}=\left(\begin{array}{cccc}
\frac{-d}{J} & 0 & 0 & 0 \\
a^{2} & -2 a & -a^{2} & 0 \\
0 & 1 & 0 & 0 \\
0 & 0 & 0 & 0
\end{array}\right), \\
A_{1}=\bar{A}_{-1}=\left(\begin{array}{cccc}
0 & 0 & 0 & \frac{1}{J j} \\
0 & 0 & 0 & 0 \\
0 & 0 & 0 & 0 \\
0 & K & 0 & 0
\end{array}\right),
\end{gathered}
$$

and $A_{m}=0$ for $|m|>1$. Similarly, $B(t), C(t)$ and $D(t)$ can also be expanded in Fourier series but since they are constant matrices, this boils down to $B_{0}=B, C_{0}=C$ and $D_{0}=D$, respectively. Next, (7) is expanded into these Fourier series:

$$
\begin{aligned}
0 & =\sum_{n \in \mathbb{Z}}\left\{s_{n} x_{n}-\sum_{m \in \mathbb{Z}} A_{n-m} x_{m}-\sum_{m \in \mathbb{Z}} B_{n-m} u_{m}\right\} e^{s_{n} t}, \\
0 & =\sum_{n \in \mathbb{Z}}\left\{y_{n}-\sum_{m \in \mathbb{Z}} C_{n-m} x_{m}-\sum_{m \in \mathbb{Z}} D_{n-m} u_{m}\right\} e^{s_{n} t} .
\end{aligned}
$$

According to the method of harmonic balance, the complex exponentials $\left\{e^{j n \omega_{p} t} \mid n \in \mathbb{Z}\right\}$ form an orthonormal basis in $L_{2}[0, T]$. Therefore, the terms enclosed by the braces must vanish and for $\forall n \in \mathbb{Z}$ there holds:

$$
\begin{aligned}
s_{n} x_{n} & =\sum_{m \in \mathbb{Z}} A_{n-m} x_{m}+\sum_{m \in \mathbb{Z}} B_{n-m} u_{m}, \\
y_{n} & =\sum_{m \in \mathbb{Z}} C_{n-m} x_{m}+\sum_{m \in \mathbb{Z}} D_{n-m} u_{m} .
\end{aligned}
$$

This system can be transformed into a Harmonic state space model. The Fourier coefficients (16-17) are stacked into an infinite Toeplitz block matrix. Because $A(t)$ covers only three non-zero Fourier coefficients, the Toeplitz matrix becomes tridiagonal:

$$
\mathcal{A}=\left(\begin{array}{ccccccc}
\ddots & \vdots & \vdots & \vdots & \vdots & \vdots & \\
\ldots & A_{0} & A_{-1} & 0 & 0 & 0 & \ldots \\
\ldots & A_{1} & A_{0} & A_{-1} & 0 & 0 & \ldots \\
\ldots & 0 & A_{1} & A_{0} & A_{-1} & 0 & \ldots \\
\ldots & 0 & 0 & A_{1} & A_{0} & A_{-1} & \ldots \\
\ldots & 0 & 0 & 0 & A_{1} & A_{0} & \ldots \\
& \vdots & \vdots & \vdots & \vdots & \vdots & \ddots
\end{array}\right)
$$

A similar definition holds for $\mathcal{B}$ in terms of $B_{0}=B, \mathcal{C}$ in terms of $C_{0}=C$, and $\mathcal{D}$ in terms of $D_{0}=D$. Altogether, this yields the harmonic state space model with infinite dimensional matrices [15]:

$$
\begin{aligned}
s \mathcal{X} & =(\mathcal{A}-\mathcal{N}) \mathcal{X}+\mathcal{B U} \\
\mathcal{Y} & =\mathcal{C} \mathcal{X}+\mathcal{D} \mathcal{U}
\end{aligned}
$$

and with an infinite vector representation for the harmonics of the state

$$
\mathcal{X}=\left[\ldots, x_{-2}^{\top}, x_{-1}^{\top}, x_{0}^{\top}, x_{1}^{\top}, x_{2}^{\top}, \ldots\right]^{\top},
$$


and similarly for input $\mathcal{U}$, and output $\mathcal{Y}$. The modulation frequency matrix $\mathcal{N}$ is defined as

$$
\mathcal{N}=\operatorname{blockdiag}\left\{j n \omega_{p} I\right\} ; \quad \forall n \in \mathbb{Z},
$$

with $I$ the identify matrix of appropriate dimension.

Definition 2 (Harmonic Transfer Function [15]): The Harmonic Transfer Function (HTF) $\mathcal{H}(s)$ describes the relationship between the harmonics of the input signal $\mathcal{U}$ and output signal $\mathcal{Y}$, such that

$$
\mathcal{Y}=\mathcal{H}(s) \mathcal{U}
$$

where

$$
\mathcal{H}(s)=\mathcal{C}[s \mathcal{I}-(\mathcal{A}-\mathcal{N})]^{-1} \mathcal{B}+\mathcal{D} .
$$

Note that the HTF acts as a generalized form of the classical describing function method [7]. A describing function is restricted to the fundamental harmonic frequency, while the HTF expresses the input-output mapping for all harmonics active in the system.

\section{HARMONIC ANALYSIS APPROXIMATION}

Direct application of the HTF is hindered as infinite sums need to be computed. Nevertheless, all linear components in Fig. 2 are proper. This allows to approximate the HTF with a truncated version $\hat{\mathcal{H}}_{N}(s)$. Only $N$ positive harmonics (as well as $N$ negative harmonics and the zero harmonic) are included in the corresponding finite state space model (21), with finite dimension for the Toeplitz block matrices $\mathcal{A}, \mathcal{B}$, $\mathcal{C}$ and $\mathcal{D}$.

Owing to the structure of $\mathcal{A}$, it turns out that $\hat{\mathcal{H}}_{N}(s)$ becomes a checkerboard with entries $\mathcal{H}_{m, n}=0$ for $m+$ $n=$ even. For $N=2$ this looks like

$$
\hat{\mathcal{H}}_{N}(s)=\left(\begin{array}{ccccc}
0 & \mathcal{H}_{-2,-1} & 0 & \mathcal{H}_{-2,1} & 0 \\
\mathcal{H}_{-1,-2} & 0 & \mathcal{H}_{-1,0} & 0 & \mathcal{H}_{-1,2} \\
0 & \hat{\mathcal{H}}_{0,-1} & 0 & \hat{\mathcal{H}}_{0,1} & 0 \\
\mathcal{H}_{1,-2} & 0 & \mathcal{H}_{1,0} & 0 & \mathcal{H}_{1,2} \\
0 & \mathcal{H}_{2,-1} & 0 & \mathcal{H}_{2,1} & 0
\end{array}\right)
$$

Physically, the structure of $\mathcal{H}(s)$ originates from (de-) modulation in the measurement system. Through the multiplication with a sinusoidal signal, all harmonics shift up and down with the fundamental frequency, see Fig. 3. By evaluating the loop at $\lambda$, it can be seen that the even terms $\omega=0,2 a, \ldots$ (arising from the applied input signal $\Delta P$ ) are modulated to odd harmonics in $\Delta f$ and demodulated back to even terms in $z$. Conversely, the odd terms $\omega=$ $a, 3 a, \ldots$ at $\lambda$ originate from the constant engine speed $w_{V}$ and are modulated to even harmonics in $\Delta f$ and return to the odd terms in $z$. As supported by $\hat{\mathcal{H}}_{N}(s)$, all signals can be simultaneously present in the system, but there is no interaction between both groups and both sets behave orthogonal.

The transfer functions $\mathcal{H}_{m, n}(j \omega)$ map an EMP input into an EMP output according to

$$
\mathcal{H}_{m, n}(j \omega): u_{n} \rightarrow y_{m}
$$

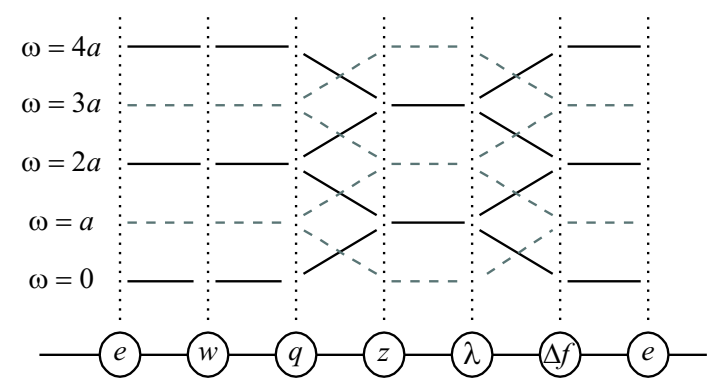

Fig. 3. Signal mappings through (de-)modulation

with the frequency range of interest given by

$$
\omega \in \Omega_{0}=\left[-\frac{a}{2}, \frac{a}{2}\right] .
$$

These transfer functions will be used to analyse the closedloop gain and the speed of convergence in Section VI.

\section{NUMERICAL STABILITY}

To prove stability for the measurement system, the wellknown Floquet Theory can be applied. It states that every (autonomous) LTP system can be reduced to a Linear Time Invariant (LTI) system via a stability preserving coordinate transformation. This way, stability for the LTP system is equivalent to that of the LTI system.

The difficulty with the Floquet theory is finding an analytical expression for the coordinate transformation matrix. At this point, literature provides several solution directions. For example, Van der Kloet [14] proposes a Floquet decomposition by applying a Riccati transform. However, this requires an explicit solution of the Riccati differential equation which is not straight forward. Instead of searching for an analytical description, one can also rely on a numerical approximation, see e.g., [3], [16].

For the work presented here, the method from Friedmann et al [3] is adopted. The state transition matrix $\Phi\left(t, t_{0}\right)$ of the LTP system (7) is numerically approximated over exactly one period $T$, using a fourth order Runge-Kutta scheme with Gill coefficients. This yields the transition matrix $\Phi\left(t_{0}+T, t_{0}\right)$. At this point it is important to select sufficient grid points $M$, to keep the step size $\Delta t=T / M$ small. Now stability of the LTP system is determined by the eigenvalues of $\Phi\left(t_{0}+T, t_{0}\right)$, which are known as the Floquet Characteristic Multipliers (FCM). The LTP system is asymptotically stable iff all FCMs appear in the open unit disk. Numerical results for this approximation can be found in the next section.

\section{Simulation RESUltS}

The preliminary simulation results presented in this section are related to the LTP system shown in Fig. 2. The selected model parameters are listed in Table I. The equivalent inertia $J$ corresponds to a mid-sized vehicle travelling at a constant speed of $50[\mathrm{~km} / \mathrm{h}]$. For convenience, the damping $d$ is chosen to be zero. The reduced system in Fig. 2 uses $K_{I C E}=K_{G e n}=1$, so $\lambda$ should converge to $\hat{\lambda}=1$. 
TABLE I

PARAMETER SETTINGS VEHICLE MODEL

\begin{tabular}{clcc} 
Symbol & Quantity & Value & Unit \\
\hline$J$ & Equivalent vehicle inertia & 24 & {$\left[\mathrm{kgm}^{2}\right]$} \\
$d$ & Friction coefficient & 0 & {$[\mathrm{Nms} / \mathrm{rad}]$} \\
$a$ & Harmonic frequency & $20 \pi$ & {$[\mathrm{rad} / \mathrm{s}]$} \\
\hline
\end{tabular}
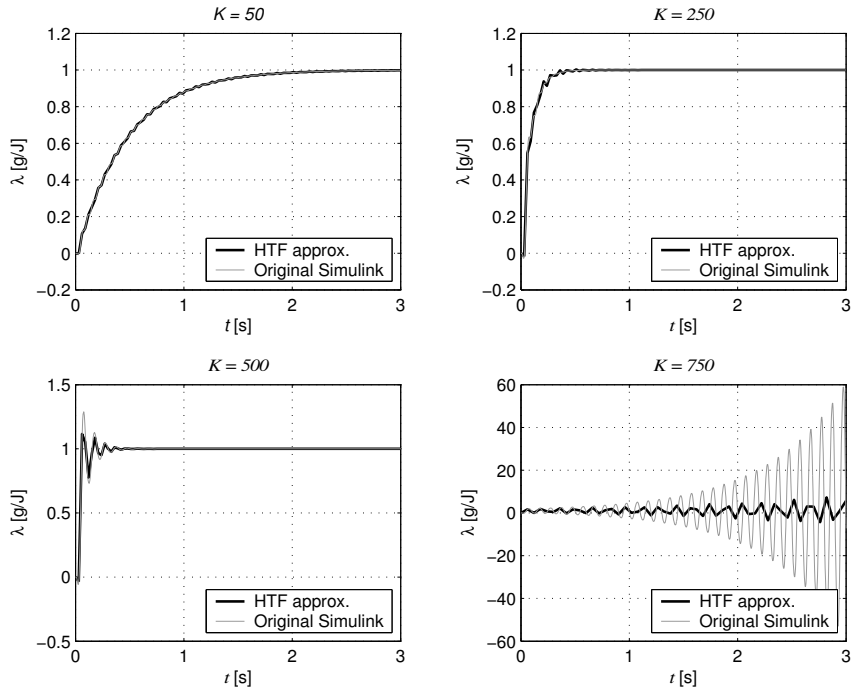

Fig. 4. Response $\lambda(t)$ for $K \in\{50,250,500,750\}$

Simulations are executed in Matlab/Simulink with different gains $K$ for controller $H_{C}(s)$. For the reference input $r(t)$, a sinusoidal signal is selected: $r(t)=2 \sin (a t), t \geq 0$. The output $\lambda(t)$ is drawn in Fig. 4. It turns out that the system exhibits non-minimum phase behavior and instability occurs for $K>714.77$.

A truncated HTF is used to approximate the simulation results from above. The HTF from (26) is calculated with $N=2$. This means that only the first two harmonics are included although the order of the non-zero entries from $\hat{\mathcal{H}}_{2}(s)$ equals 7. Again, the sinusoidal reference signal $r(t)=$ $2 \sin (a t)$ is studied. With $r(t)=0$ for $t<0$, its Laplace transform is equal to $R(s)=\frac{2 a}{s^{2}+a^{2}}$. This signal is applied to $\hat{\mathcal{H}}_{2}(s)$ :

$$
\hat{\mathcal{Y}}_{2}(s)=\hat{\mathcal{H}}_{2}(s) \hat{\mathcal{U}}_{2}(s)
$$

with

$$
\hat{\mathcal{Y}}_{2}(s)=\left[\begin{array}{l}
Y(s-2 a) \\
Y(s-1 a) \\
Y(s) \\
Y(s+1 a) \\
Y(s+2 a)
\end{array}\right] ; \hat{\mathcal{U}}_{2}(s)=\left[\begin{array}{l}
R(s-2 a) \\
R(s-a) \\
R(s) \\
R(s+a) \\
R(s+2 a)
\end{array}\right] .
$$

From all harmonic signals in $\hat{\mathcal{Y}}_{2}(s)$, especially $Y(s)$ is of interest. This signal has been isolated and for different values of $K$, the time response is also shown in Fig. 4.

Comparing the simulation results with the HTF, it can be seen that $N=2$ yields a good approximation for the original LTP system, provided that $K$ is sufficiently small. However,
TABLE II

\begin{tabular}{ll}
\multicolumn{2}{c}{ NUMERICAL STABILITY } \\
Model & Stability region \\
\hline Simulink model & $K \leq 714.78$ \\
HTF approximation & $K \leq 733.61$ \\
FCM approximation & $K \leq 714.05$ \\
\hline
\end{tabular}

for $K$ close to or above the instability point, differences in amplitude emerge.

From the results in Fig. 4 it is also clear that the selected gain $K$ has a significant influence on the performance of the measurement system. When a small gain is selected, the system suffers from a large settling time, but there is no overshoot. Conversely, a large gain yields a faster response but it is accompanied by overshoot. Good results are obtained when the system is critically damped with $K=390$. A moderate value for $K$ also preserves stability in practical situations when $K_{I C E}$ changes over time.

The influence of noise is briefly considered by adding a band-limited white noise signal $\psi$ to the input signal $r(t)$. The amplitude of the noise spectrum is defined as:

$$
\Psi(\omega)= \begin{cases}1.0 \times 10^{-3} & \text { for }|\omega| \leq 1000 \pi \mathrm{rad} / \mathrm{s} \\ 0 & \text { for }|\omega|>1000 \pi \mathrm{rad} / \mathrm{s}\end{cases}
$$

This signal has a serious impact on the input signal $r(t)=$ $2 \sin (a t)+\psi$, as can be seen in Fig. 5. Nevertheless, the disturbance on $\lambda$ remains limited to a zero-mean slow varying signal. This means that measuring over a longer time period is sufficient to obtain a correct estimate for $\lambda$. This method will not work if dominant noise is present around harmonic frequency $a$. In this case, $\lambda(t)$ suffers from an offset error. An offset can also be created by signals passing through the loop more than once. For example disturbances at odd harmonics of $a($ i.e. $3 a, 5 a, \ldots)$ in the input signal $r(t)$ will cause an offset for $\lambda(t)$.

Numerical stability of the system is considered in three ways. First, the Simulink model is used to determine the value for $K$ which leads to instability. Next, the truncated HTF from Section IV is used to verify if similar results emerge. Finally, the transition matrix $\Phi\left(t_{0}+T, t_{0}\right)$ is numerically calculated using $M=1000$ steps for period $T$. The FCMs from Floquet Theory are computed to determine stability of the system. The corresponding results are summarized in Table II.

According to the results in Table II, the stability bound estimated by the HTF is too optimistic. It follows that the omitted higher order harmonics have still some influence in the approximated HTF. The numerical approximation of the FCMs yields excellent results to determine stability.

\section{DISCUSSION}

The measurement system only uses one frequency $a$ for identification of $\lambda$. Consequently, the applied method is only valid if $\lambda$ is not frequency dependent $(=\lambda(a)$ constant $)$. This needs to be proven by experimental data. The advantage of only using frequency $a$ for signal correlation is that the model 

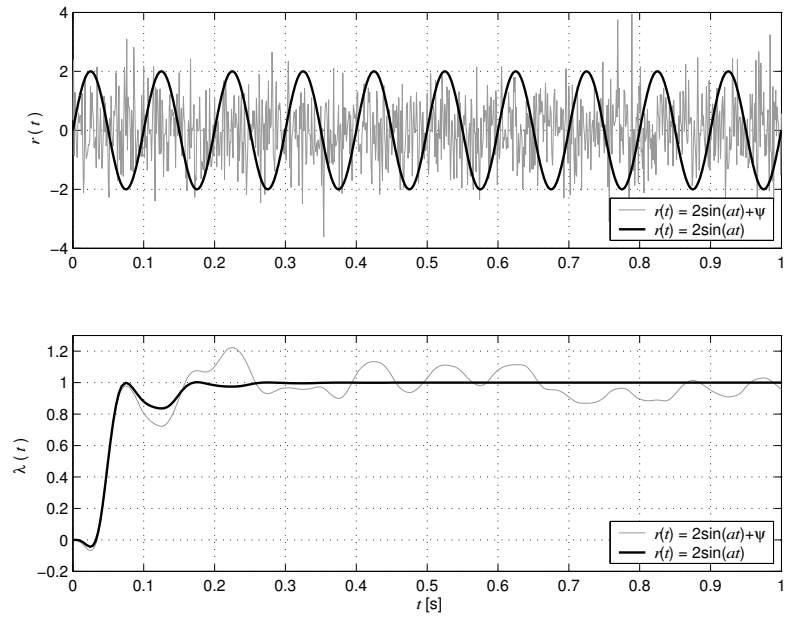

Fig. 5. Response $\lambda(t)$ with additive noise and $K=390$

needs to be accurate only at this frequency, whereas model errors at other frequencies will not degrade the quality of $\lambda$.

The results in Fig. 4 indicate that the selected gain $K$ influences the speed of convergence of the measurement system. Eventually, the speed of convergence is limited by the characteristics of the band-pass filter $H_{F}(s)$. This filter introduces two poles with time constant equal to $\tau=$ $2 \pi / a=0.1$ [s]. The filter design is selected according to the intended input signal $r(t)$, so it follows that a higher harmonic frequency $a$ can lead to a faster convergence speed.

Unfortunately, selecting a higher harmonic frequency leads to more attenuation in the measurement system, due to the low-pass characteristics of $H_{V}(s)$. This can be compensated by a higher gain $K$, but small variations for the engine speed are difficult to measure. Moreover, frequency $a$ should always be sufficiently low compared to the dynamics of the ICE and the generator, to allow for static gains when modelling these components.

To select a suitable value for the harmonic frequency $a$, also the influence of sensor noise needs to be further analyzed. This will be a topic for future research. This research should also consider the current filter design for $H_{F}(s)$. Possibly other filters exist to optimize the system for better disturbance rejection.

Another method to improve the speed of convergence, is by using old data as an initial guess. Starting from a well defined initial value, conversion towards the final value becomes possible within 0.1 [s]. This result offers possibilities to apply the measurement system under many operating conditions, e.g., in transient situations where the vehicle is accelerating or decelerating, but also during gear changes, or after a cold start of the ICE and analyse the impact of the engine temperature on $\lambda$.

\section{Conclusions}

By recognizing the wish for accurate model parameters for an EM system, this paper presents a novel measurement system to estimate the fuel cost for generating electric power on-line in the vehicle. A correlation technique is introduced covering two multiplications with a sinusoidal signal. With this measurement scheme it is possible to equip existing EM strategies with accurate values of the incremental fuel cost. Since this parameter gets updated along the vehicle life cycle, vehicle wear is automatically diagnosed.

It is shown that the control scheme satisfies the class of LTP systems. This allows Harmonic Analysis to investigate the dynamic behavior of the proposed measurement system. An analytical transfer function is found which approximates the LTP system in most situations. Stability bounds are more accurately calculated by Floquet theory.

Simulation results illustrate how to optimize the speed of convergence, by selecting a suitable gain for the controller. These results also show robustness of the measurement system against noise on the applied excitation signal.

\section{REFERENCES}

[1] J.H. Buckland and J.A. Cook. Automotive emissions control. In Proc. of the American Control Conf., pages 3290-3295, Portland, OR, June 2005.

[2] C.C. Chan. The state of the art of electric, hybrid, and fuel cell vehicles. Proceedings of the IEEE, 95(4):704-718, April 2007.

[3] P. Friedmann, C.E. Hammond, and Tze-Hsin Woo. Efficient numerical treatment of periodic systems with application to stability problems. International Journal for Numerical Methods in Engineering, 11(7):1117-1136, 1977.

[4] L. Guzzella and A. Sciarretta. Vehicle Propulsion Systems - Introduction to Modeling and Optimization. Springer-Verlag, Berlin Heidelberg, 2005.

[5] J.T.B.A. Kessels. Energy Management for Automotive Power Nets. $\mathrm{PhD}$ thesis, Department of Electrical Engineering, Technische Universiteit Eindhoven, The Netherlands, 2007.

[6] J.T.B.A. Kessels, M. Koot, B. de Jager, P.P.J. van den Bosch, N.P.I. Aneke, and D.B. Kok. Energy management for the electric powernet in vehicles with a conventional drivetrain. IEEE Trans. on Control Systems Technology, 15(3):494-505, May 2007.

[7] H.K. Khalil. Nonlinear Systems. Prentice Hall, Upper Saddle River, $3^{\text {rd }}$ edition, 2002.

[8] D. Khiar, J. Lauber, T. Floquet, and T.M. Guerra. An observer design for the instantaneous torque estimation of an IC engine. In Proc. of the IEEE Vehicle Power and Propulsion Conference, pages 391-395, Chicago, IL, September 2005.

[9] P. Pisu and G. Rizzoni. A comparative study of supervisory control strategies for hybrid electric vehicles. IEEE Trans. on Control Systems Technology, 15(3):506-518, May 2007.

[10] G. Rizzoni and Y. Zhang. Identification of a non-linear internal combustion engine model for on-line indicated torque estimation. Mechanical Systems and Signal Processing, 8(3):275-287, May 1994.

[11] A. Sciarretta, L. Guzzella, and M. Back. A real-time optimal control strategy for parallel hybrid vehicles with on-board estimation of the control parameters. In Proc. of the IFAC Symp. on Advances In Automotive Control, Salerno, Italy, April 19-23 2004.

[12] J. Shen, A. Masrur, V.K. Garg, and J. Monroe. Automotive electric power and energy management: A system approach. In Business Briefing: Global Automotive Manufacturing and Technology. Touch Briefings, London, UK, April 2003.

[13] Ying Tan, Dragan Nešić, and Iven Mareels. On non-local stability properties of extremum seeking control. Automatica, 42(6):889-903, June 2006.

[14] P. van der Kloet. Modal solutions for linear time-varying systems. PhD thesis, Technische Universiteit Delft, The Netherlands, 2002.

[15] N.M. Wereley and S.R. Hall. Frequency response of linear time periodic systems. In Proc. of the 29th IEEE Conf. on Decision and Control, pages 3650-3655, Honolulu, Hawaii, December 1990.

[16] J. Zhu and S.K. Vemula. A new method for evaluating floquet characteristic exponents of periodic linear systems. In Proc. of the American Control Conf., pages 221 - 225, San Francisco, CA, June 1993. 